\title{
Kerr effect in structured superluminal media
}

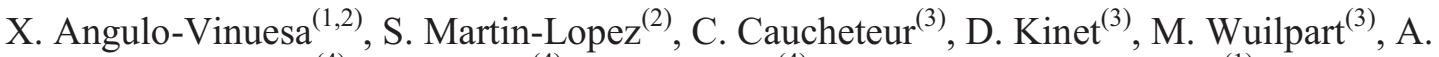 \\ Denisov $^{(4)}$, S. H. Chin ${ }^{(4)}$, L. Thévenaz ${ }^{(4)}$ and M. Gonzalez-Herraez ${ }^{(1)}$ \\ ${ }^{(1)}$ Departamento de Electrónica, Universidad de Alcalá, Escuela Politécnica DO-231. \\ 28871 Madrid, Spain. E-mail: miguelg@depeca.uah.es \\ ${ }^{(2)}$ Instituto de Óptica, Consejo Superior de Investigaciones Científicas. Madrid, Spain. \\ (3) Electromagnetism and Telecommunication Department, Faculté Polytehnique, Université \\ de Mons, Belgium \\ ${ }^{(4)}$ Group for Fiber Optics, Ecole Polytechnique Federale de Lausanne, Switzerland.
}

\begin{abstract}
Kerr effect accounts for the change in refractive index of a material with the light intensity and appears in all known optical materials. In this work we analyze Kerr effect in structured superluminal media (e.g some specific types of resonators). We show that Kerr effect in these structures can be cancelled or even reversed (in comparison with the Kerr effect of the material composing the structure) depending on the group index of the structure. We also discuss some possible realizations of structured superluminal media.
\end{abstract}

Keywords: slow light, linear systems, nonlinear regeneration.

\section{INTRODUCTION}

Along the last years, the focus of slow light research $[1,2]$ has shifted from the creation of delay lines to the enhancement of light-matter interactions. Light-matter interactions are weak in most optical media in conventional conditions. Slow light structures allow a large reduction of the group velocity of the light signals travelling through them, and, as a consequence, a strong confinement of the electromagnetic field and a great enhancement of light-matter interactions. These interactions include various linear and nonlinear effects (including Kerr effect) as well as spontaneous emission. In the particular case of Kerr effect (change in refractive index with light intensity), the combination of longer transit times and higher power densities increase the efficiency of the effect by a factor proportional to the square of the group index. For example, many photonic crystal waveguides can exhibit group index values close to 100 over $1 \mathrm{THz}$ bands. In these waveguides, one may expect Kerr effect enhancement factors up to 10000 over this bandwidth, which is extremely remarkable. Such enhancement factors are impossible to achieve otherwise (e.g. engineering of the waveguide effective area). As a result of this new approach, one can envisage many devices arising from the possibility of doing practical (low-power) nonlinearities in conventional materials and fabrication processes.

While this interest in slow light structures has been thoroughly discussed, there has been little or no discussion on the possibility of actually cancelling Kerr effect in superluminal media. Kerr effect cancellation is interesting from a technological point of view since all known optical material exhibit Kerr effect. While, for typical applications, Kerr effect is negligible, in some applications it turns out to have a detrimental impact. As an example, the precision of fiber-optic gyroscopes is known to be severely impaired by Kerr effect. Fiberoptic gyroscopes rely on optical fibers, which show usually a positive Kerr coefficient, this is, the refractive index grows with the intensity. The basic structure of these devices relies on a Sagnac interferometer. Non-

Advances in Slow and Fast Light V, edited by Selim M. Shahriar, Frank A. Narducci, Proc. of SPIE Vol. 8273, 827310 - @ 2012 SPIE · CCC code: 0277-786X/12/\$18 · doi: 10.1117/12.915287 
reciprocal phase changes owing to rotation are recorded at the output of the device as a change in the transmission, and any reciprocal perturbation (temperature, etc) is cancelled by the structure itself. One of the main sources of error in these artifacts is that the coupling ratio of the coupler needed to develop the gyroscope never has an exact ratio of 50\%. This carries as a consequence that the counter-propagating signals show different power levels. Due to the Kerr effect, this imbalance causes a non-reciprocal phase offset which is proportional to the power difference. Since the coupling ratio slightly varies, through the effect of e.g. temperature variations, the offset varies too, which at the end induces an indistinguishable signal variation from a rotation-induced signal. Air-core fibers have been proposed to solve this issue [3], however they introduce additional errors due to their imperfect coupling properties with conventional single-mode fibers.

Using conventional perturbation theory [4], it can be shown that Kerr effect can be cancelled in media exhibiting $n_{g}=0$, and reversed when $n_{g}<0$. In this paper we discuss this possibility as well as possible structures that would allow achieving this situation. In particular, we will describe the observation of tunable superluminality in two types of fiber-optic based structures: phase-shifted chirped fiber Bragg gratings and lossy ring resonators. We will show, both theoretically and experimentally, that these two structures can exhibit group index values of zero or below over relatively small bandwidths. Furthermore, we will show that Kerr effect in these structures can indeed be cancelled or reversed over a certain power range.

\section{KERR EFFECT IN STRUCTURED SLOW/FAST LIGHT MEDIA}

Kerr effect in slow/fast light structures can be analyzed using simple perturbation theory [4]. This can be justified considering that Kerr effect generally causes extremely small refractive index changes. In this scenario, it can be shown that the effect of any refractive index change $(\Delta n)$ can be accounted for by a corresponding shift in the dispersion relation, hence $\omega(k) \rightarrow \omega(k)+\omega_{s} \Delta n / n$, where $\omega_{s}$ is the carrier frequency, considered given in normal experimental conditions. The corresponding phase change caused at the carrier frequency can be simply derived from the deviation of the dispersion curve $\Delta \phi=d k / d \omega \cdot\left(\omega_{s} \Delta n / n\right) \cdot L=\omega_{s} \Delta n L / n v_{g}$. It is thus clearly visible that any refractive index change in these structures causes a phase change that is affected by a factor proportional to the group index. The origin of the quadratic enhancement of Kerr effect with the group index lies in the term $\Delta n$ itself, which is also enhanced by a factor proportional to $n_{g}$ (the pulse in a slow light medium is spatially compressed, leading to higher peak intensities by a factor of $n_{g}$ ).

It is then clear that high group indexes allow strong enhancements of the Kerr effect, a possibility that offers a large potential for the development of all-optical devices. This possibility has been largely commented upon in the literature. However no comment has been done for the situations in which $n_{g}$ falls below one or even below zero. In these conditions, the previous analysis also holds, leading to zero Kerr effect for $n_{g}=0$ or reversed Kerr effect for $n_{g}<0$. The possibility of actually cancelling the Kerr effect in an optical material is rather exciting, since all known optical materials exhibit Kerr effect. Although these conditions offers far less possibilities than the situation of large group index, it is both interesting from a fundamental point of view and also from a technological perspective. It is interesting to note that this group index dependence appears only in those cases known as structural slow light media (photonic crystals, resonators, etc). Kerr effect in material slow light media does not follow the same rules. In the next sections we will show, both theoretically and experimentally, different fiber-based structures that exhibit fast light and that seem suitable for Kerr effect cancellation.

\section{FAST LIGHT IN PHASE-SHIFTED CHIRPED FIBER BRAGG GRATINGS}

In this section we demonstrate experimentally that a wide range tuning of the group delay of a chirped Fiber Bragg Grating (CFBG) is possible, including situations of superluminal propagation. It is based on the 
creation of a temporary phase-shift introduced locally by a wire heater [5]. This setup is particularly attractive for the implementation of simultaneously wavelength and group index-tuneable devices. The phase-shift can be accurately controlled through the current applied on the wire and the wavelength can be tuned by moving the wire along the CFBG. Another remarkable feature of this setup is the capability to switch between strong delay or advancement depending on the phase-shift value. Using a $10 \mathrm{~cm}$ long CFBG, we report experimentally delay/advancement of more than $1000 \mathrm{ps}$ in a frequency range of a few GHz. Although it is difficult to determine the exact group index achieved (since the reflection is distributed), this delay variation far exceeds the maximum delay corresponding to the physical length of the device, which, in the case of advancement, implies group index values below 0 .

Our experiments were realized on a $10 \mathrm{~cm}$-long linearly chirped FBG written into a hydrogen-loaded singlemode fiber through a $1070.040 \mathrm{~nm}$-period phase mask characterized by a chirp rate of $49 \mathrm{pm} / \mathrm{cm}$. As shown in Fig.1, the CFBG reflection spectrum presents a $3 \mathrm{~dB}$ bandwidth of $0.71 \mathrm{~nm}$ while its dispersion is around $1400 \mathrm{ps} / \mathrm{nm}$. The grating is not apodized, which explains the strong ripple on the group delay curve in the reflection band. All measurements were carried out using the optical vector analyzer CTe from Luna Technologies with a $3 \mathrm{pm}$ wavelength resolution and a wavelength sampling set to $10 \mathrm{pm}$. In the setup, a $\mathrm{NiCr}$ wire heater (diameter of about $0.2 \mathrm{~mm}$ ) is placed perpendicularly to the stripped CFBG. A voltage source and a variable resistor are used to control the temperature of the wire. Because of the thermo-optic effect, the refractive index of the fiber locally changes with the temperature of the wire heater. As a result, a phase shift is introduced in this region of the grating. Our experiments have revealed that a temperature elevation of about $150{ }^{\circ} \mathrm{C}$ yields a $\pi$ phase shift.

Figure 2 depicts the reflected amplitude and group delay evolutions when a $0.2 \mathrm{~mm}$-wide region of the grating is heated. Figure 2(a) presents the results obtained for a phase-shift slightly smaller than $\pi$. A notch of $\sim 15 \mathrm{~dB}$ is induced in the spectrum at the phase-shift location. It results in a strong additional delay of $\sim 1200 \mathrm{ps,} \mathrm{which}$ is about 200 ps higher than the maximum delay offered by the used CFBG. Fig. 2(b) depicts the results for a phase-shift slightly above $\pi$. A negative variation of the delay reaching more than $1000 \mathrm{ps}$ is induced in this case. Hence, at the same wavelength, a flip-flop between sub- and superluminal propagation is achievable in the notch. In both cases, the bandwidth of the notch is $\sim 5 \mathrm{GHz}$ (FWHM), which is quite common for phaseshift devices. Also, one can change the working wavelength by moving the wire along the CFBG. Thus, the system allows both wavelength and delay tuning.

Figure 3 displays the evolution of the group delay variation as a function of the phase-shift value. The latter was computed from the temperature reached around the NiCr wire using the relationship given in ref. [6]. Figure 3 clearly demonstrates the possibility to accurately tune the group delay variation, either positively or negatively. As shown in Fig. 2, the wavelength range in which the phase-shift occurs is characterized by a strong amplitude decrease. In practice, this amplitude distortion can be drastically attenuated by locally heating two closely-spaced sections of the CFBG. Indeed, thanks to the induced wavelength shift, one can use the first heated region to decrease the amplitude spectrum variation on the second one, while keeping constant the group delay variation. Figure 4 shows a comparison between a single heated configuration (Fig. 4a) and a double heated configuration (Fig. $4 \mathrm{~b}-13 \mathrm{~mm}$ of distance between heated regions) where the same delay is reached and the loss is highly reduced at the operating wavelength. The second configuration is thus more interesting to use in practice.

Finally, let us mention that all our experiments are consistent with numerical simulations (not shown here) carried out using the coupled mode equations with parameters close to those of the experimental CFBG. 


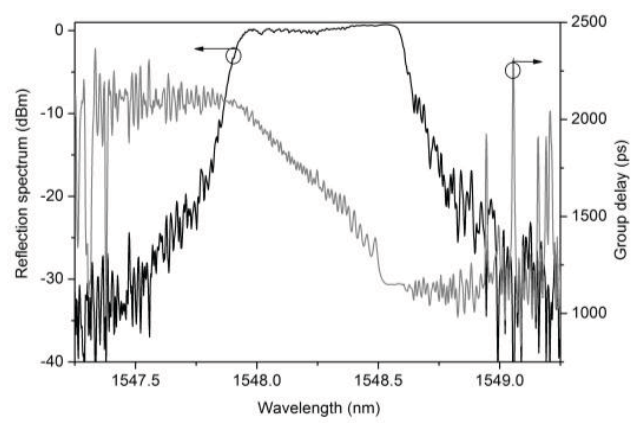

Fig. 1. CFBG reflected spectrum and associated group delay evolution (light launched through the long-wavelength port).

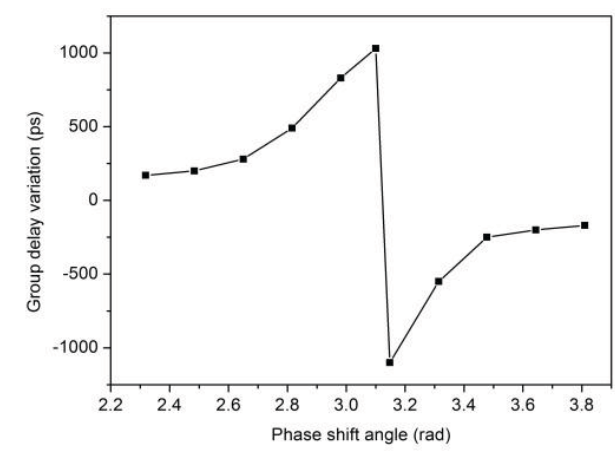

Fig. 3. Evolution of the group delay as a function of the thermally-induced phase shift value.

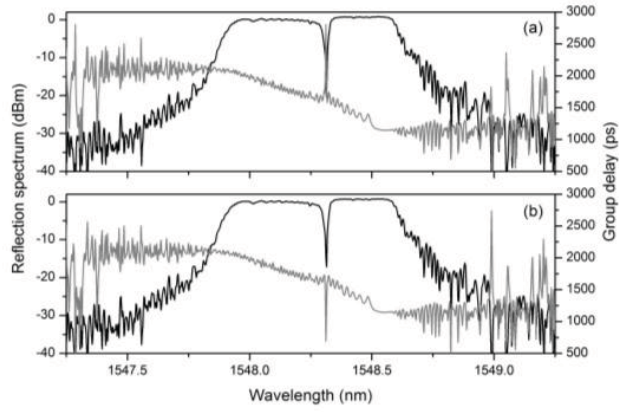

Fig. 2. Amplitude and phase spectra for a 0.2 $\mathrm{mm}$ heated region yielding a phase-shift $<\pi$ (a) and $>\pi(b)$.

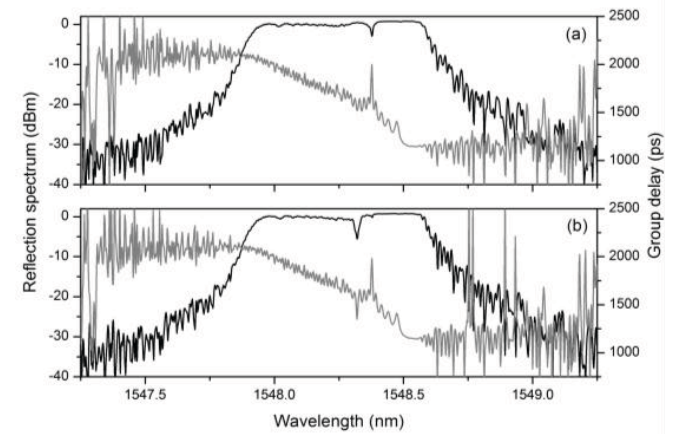

Fig. 4. Comparison between a single phase shift region (a) and two closely spaced phase shift regions (b) yielding the same delay.

\section{FAST LIGHT IN LOSSY FIBER-BASED RING RESONATORS}

In this section we perform a theoretical study of slow and fast light in lossy fiber ring resonators. We show that, depending on the coupling ratio and the losses in the resonator, the group delay of the ring resonator can be tuned from strong delay to strong advancement, including situations of negative group index. Furthermore, we also demonstrate theoretically that, when these structures are engineered to have zero group index, they also exhibit zero Kerr effect.

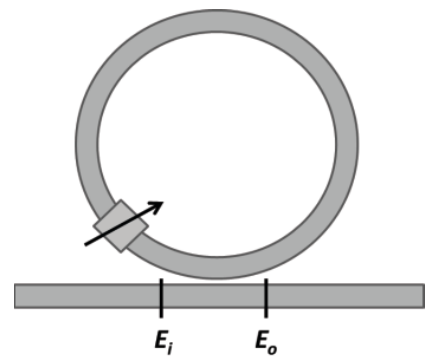

Fig.5: fiber-based ring resonator considered. Light travels from left to right. 
We consider the structure depicted in Fig. 5, i.e. the typical fiber ring resonator with a variable loss element inside. Our analysis of this structure is similar to the analysis done by Heebner et al. [7] for resonator-coupled waveguides. A transfer function can be found for the case of the lossy resonator:

$$
\frac{E_{o}}{E_{i}}=\frac{\kappa \exp \left(\frac{i \omega n L}{c}\right)-a}{\exp \left(\frac{i \omega n L}{c}\right)-\kappa a}
$$

Where $\kappa$ and $a$ are, respectively, the coupling coefficient and the losses in the resonator. As expected, this transfer function leads to resonances in the spectrum with a periodicity given by $2 \pi n L / c$. It can be easily shown that, when the losses exceed the coupling (undercoupling), negative group delays can be found at the resonances, while they remain positive in the opposite case (overcoupling). Fig. 6 displays the phase, modulus and group index response of the structure considered for the two cases mentioned, showing the different qualitative behavior between the overcoupled and undercoupled cases.
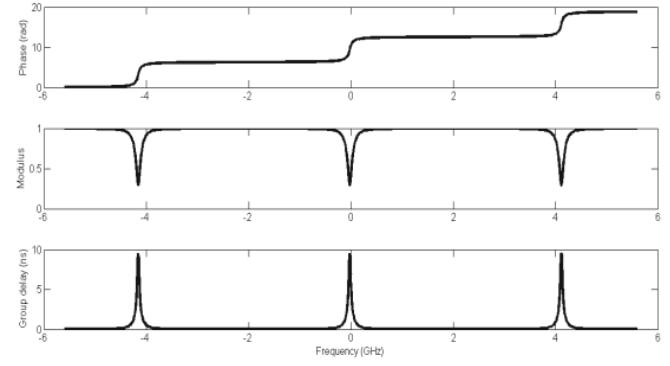

gi(a)
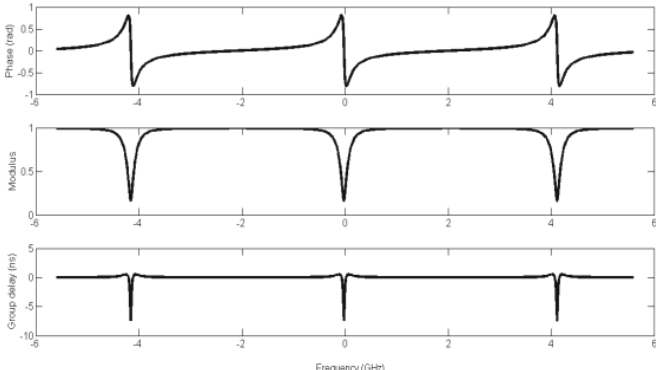

(b)

Fig.6: phase, modulus and group delay of the resonator considered. The length of the resonator is set to $5 \mathrm{~cm}$, coupling coefficient is $0.31 \mathrm{~dB}$. In (a) the losses in the resonator are tuned to $0.17 \mathrm{~dB}$ while in (b) they are set to $0.43 \mathrm{~dB}$. Refractive index is set to 1.45 . Center wavelength is $\approx 1554 \mathrm{~nm}$.

By precisely tuning the losses in the above structure one can achieve zero group delay of close to any length of fiber followed by the resonator. As an example, we consider a section of $20 \mathrm{~cm}$ of fiber followed by the same resonator as in Fig. 6, in which the losses have been tuned to 0.82 dB. Fig. 7 shows the phase, modulus and group index of the structure considered, showing zero group delay over the resonance. The dashed line shows the same for a structure in which there has been an index elevation of $10^{-7}$, a typical value that could be achieved in a fiber through an intense beam. As it can be seen, there is a shift of the global phase response in these conditions (the FSR slightly changes by the same amount), but overall the response of the structure remains a zero phase change at the wavelengths of zero group delay, which is consistent with the above reasoning. Of course this holds many restrictions (it is only valid for a certain wavelength range), but it proves that Kerr effect can be cancelled through an intelligent structuration of the material.

\section{CONCLUSIONS}

We have shown that structured superluminal media can be engineered to show zero Kerr effect. We have shown two structures that exhibit fast light through plain structuration (not through material preparation). The 
implications of this are basic as well as technological, since all known optical media exhibit Kerr effect. Experimental demonstration of these effects is underway.
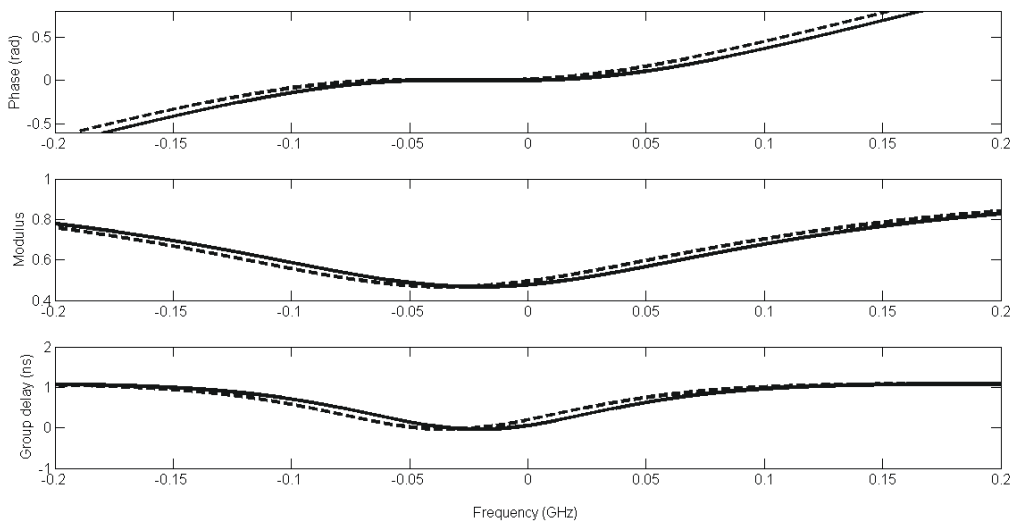

Fig.7: phase, modulus and group delay of the resonator considered, after a $20 \mathrm{~cm}$ piece of fiber. The losses in the resonator are tuned to $0.82 \mathrm{~dB}$.

\section{ACKNOWLEDGMENTS}

We acknowledge financial support from the Ministerio de Educacion y Ciencia through projects TEC200914423-C02-02, and the support from the Comunidad Autónoma de Madrid through the project FACTOTEM2 S2009/ESP-1781.

\section{REFERENCES}

[1] R. W. Boyd and D.J. Gauthier, "'Slow' and 'Fast' light," Ch. 6 in Progress in Optics 43, E. Wolf, Ed. (Elsevier, Amsterdam, 2002), 497-530.

[2] J. B. Khurgin and R. S. Tucker, Slow light: Science and applications (CRC Press, Boca Raton, 2009).

[3] V. Dangui, M. J. F. Digonnet, and G. S. Kino, "Laser-driven photonic-bandgap fiber optic gyroscope with negligible Kerr-induced drifts," Opt. Lett., vol. 34, 875-877, 2009.

[4] M. Soljacic, S. G. Johnson, S. Fan, M. Iban escu, E. Ippen, and J. D. Joannopoulos, "Photonic-crystal slow-light enhancement of nonlinear phase sensitivity," J. Opt. Soc. Am. B, vol. 19, 2052, 2002.

[5] C. Caucheteur, D. Kinet, A. Mussot, A. Kudlinski, P.Megret and M. Gonzalez-Herraez, "Group Delay Tuning in Phase-Shifted Chirped FBGs," Proc. of the OSA Bragg Grating Photosensitivity and Poling in Glass Waveguides Topical Meeting, BThB4, 2010. See also Photonics Technology Letters (to appear)

[6] M. Li, H. Li, Y. Painchaud, "Multi-channel notch filter based on a phase-shifted phase-only-sampled fiber Bragg grating," Opt. Expr. Vol. 16, 19388-19394, 2008.

[7] J. Heebner and R. W. Boyd "Slow and fast light in resonator-coupled waveguides" J. Mod. Optics vol. 49 26292636,2002 